\title{
Perforation Studies on Flat Bars for XFEM-Based Failure Analysis
}

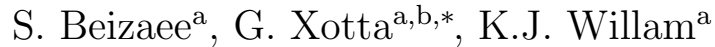 \\ ${ }^{a}$ Department of Civil and Environmental Engineering, Cullen College of Engineering, \\ University of Houston, N107 Engineering Building 1, Houston TX 77204-4003, USA. \\ E-mail addresses: sbeizaee@uh.edu; kwillam@uh.edu \\ ${ }^{b}$ Department of Civil, Environmental and Architectural Engineering (DICEA), \\ University of Padova, Via F. Marzolo 9, 35131 Padova, Italy.E-mail address: \\ giovanna.xotta@dicea.unipd.it
}

\section{Abstract}

This paper revisits the stress concentration problem in the proximity of circular perforations of different diameters and presents results of an experimental program on flat bars made of high strength steel, mild steel, cast iron and aluminum alloys. A digital image correlation (DIC) system is used to monitor the experiments and provide displacement and strain fields on the surface of the specimens at different stages of axial loading. The extended finite element method (XFEM) is introduced and applied to the inelastic deformation regime by coupling localization analysis and XFEM to discretely follow crack propagation into the ductile response regime.

Keywords: Stress concentration, Localization Analysis, DIC System, IXFEM, Crack propagation

\section{Nomenclature}

a distance

\footnotetext{
${ }^{*}$ Corresponding Author, Tel.:+390498275592
} 


\begin{tabular}{|c|c|}
\hline $\mathbf{a}_{\mathbf{i}}$ & nodal enriched degree of freedom vector \\
\hline $\mathbf{b}_{\mathbf{i} \alpha}$ & nodal enriched degree of freedom vector \\
\hline $\mathbf{E}^{\mathrm{e}}$ & elastic tangent operator \\
\hline $\mathbf{E}^{\mathrm{ep}}$ & elastoplastic tangent operator \\
\hline$F$ & yield function \\
\hline$F_{\alpha}(\mathbf{x})$ & crack-tip functions \\
\hline$H(\mathbf{x})$ & Heaviside function \\
\hline$H^{p}$ & hardening modulus \\
\hline$H_{c r i t}^{p}$ & critical hardening modulus \\
\hline$J_{2}$ & second invariantof the deviatoric stress tensor \\
\hline$K$ & hardening variable \\
\hline K & stiffness tensor \\
\hline $\mathbf{m}$ & normal to the potential function \\
\hline M & polarization vector \\
\hline $\mathbf{n}$ & normal to the yield function \\
\hline $\mathbf{N}$ & normal vector to the discontinuity surface \\
\hline$N_{i}(\mathbf{x})$ & shape functions \\
\hline $\mathrm{Q}^{\mathrm{e}}$ & elastic localization tensor \\
\hline $\mathrm{Q}^{\mathrm{ep}}$ & elastoplastic localization tensor \\
\hline$r$ & polar coordinate \\
\hline s & deviatoric stress tensor \\
\hline t & nominal traction stress vector \\
\hline $\mathbf{u}$ & displacement vector \\
\hline$\gamma$ & jump across discontinuity surface \\
\hline$\delta$ & separation vector \\
\hline
\end{tabular}




$\begin{array}{ll}\boldsymbol{\epsilon} & \text { strain tensor } \\ \theta & \text { polar coordinate } \\ \theta_{c r} & \text { critical angle } \\ \lambda_{\min } & \text { minimum eigenvalue } \\ \boldsymbol{\sigma} & \text { stress tensor } \\ \sigma_{y} & \text { yield strength of the material } \\ \tau_{\max } & \text { maximum shear stress } \\ \mathbf{1} & \text { identity tensor }\end{array}$

\section{Introduction}

This paper is focused on the response behavior of brittle and ductile failure by coupling plasticity with extended finite element method in order to model discrete crack initiation and extend the initiation phase to fracture propagation. This is accomplished by exploiting appropriate crack initiation criteria and performing analytical and numerical localization analysis to determine the critical orientation of the emerging failure surface [1], [2]. In order to model the failure behavior a linear elastic material behavior is assumed up to the initial yield limit and appropriate hardening functions are used to describe the post-yield behavior in the plastic range up to peak resistance of the specimen and into the post-peak regime of specimen separation. Plasticity is used in order to account for the irreversible behavior of materials, especially ductile metals that experience large flow and plastification. To trigger plasticity during loading there has to be a yield envelope. One of the early yield conditions for metals is the Tresca hexagonal criterion [3], proposed for modeling shear failure of metal plasticity. The von Mises 
yield criterion [4] is an extension of the Tresca criterion, expressing the onset of yielding in terms of the second deviatoric stress invariant that is also used to control the plastic flow under persistent loading (Prandtl-Reuss flow rule). Besides these two yielding criteria, that are independent of hydrostatic stresses, there are several yield envelopes to account for the first invariant of stress tensor and to account for the reynolds effect of volumetric-deviatoric coupling in frictional materials such as concrete rock and soils ([5], [6], [7]). The effect of the third deviatoric invariant used in concrete [8] has also been adopted for metals in automotive industrial applications. In this work the von Mises and Tresca yield criteria are used in order to initiate crack propagation for mild steel, high strength steel and aluminum alloys. As regards the brittle cast iron, the Rankine and the St. Venant criteria are used to detect mode I failure in tension.

For quantitatively assess and validate the numerical results, a series of experiments, in uniaxial tension, were performed on mild steel, high strength steel, cast iron and aluminum flat bars and a digital image correlation (DIC) system was utilized to acquire images of the yielding and failure processes in the perforated test specimens. This technique, originally developed in the 1980's ([9], [10], [11], [12], [13]) is a high resolution photogrammetric one for full-field non-contact measurements including displacement, deformation, crack growth, and other measurements of complex test geometries. This can be achieved by processing and comparing two digital images of the specimen, captured by high resolution cameras in the reference and target states respectively. The ARAMIS DIC-3D software by GOM [14] has been adopted to process the measurements, to automatically compute field data and to per- 
form post-processing of surface deformation fields. The DIC system provides additional insight into the development of failure bands and the concomitant strain redistribution throughout the test specimens.

A well-known phenomenon in literature regarding metals is the appearance of shear bands which show the formation of weak discontinuities in form of an instability condition of the so-called localization tensor within the material, with large deformations concentrated in a small region of the structure narrow to the bands. This phenomenon, also called strain localization, is referred to the formation of discontinuity in the strain rate field and localization analysis provides a description of it. Probing the state of the material with regard to possible localization directions leads to an eigenvalue problem similar to the classical buckling analysis of slender compressive members. This involves searching for singular conditions of the so called localization or acoustic tensor, since its definition originates in wave propagation arguments. Localization analysis can be performed for any material model in form of a tangential constitutive law (indeed the localization tensor is defined by double contraction of the tangent operator with the normal vector of the possible discontinuity surface).

Localization analysis has been extensively developed and discussed in the framework of elasto-plasticity $([15],[16],[17])$; in this paper the localization diagnosis is performed analytically for the yield criteria listed above and the expression of the critical hardening modulus and critical localization directions are provided and evaluated. Localization analysis has been also extended, when the tangent stiffness is provided, to material describing stiffness degradation (elastic-fracturing, elastic-damage) and to multi-dissipative 
materials (plasticity combined with elastic degradation) by solving the localization eigenproblem comprising multiple rank-one updates ([18], [19]).

In order to avoid formation of weak discontinuities in the degrading branch and to discretely capture fracture in form of strong discontinuities, the extended finite element method (XFEM) is incorporated in the description of the degradation process. The concept of inelastic extended finite element method (IXFEM), is introduced by coupling localization analysis and extended finite element method to track the crack propagation in ductile material regimes.

The extended finite element method is a particular form of the partition of unity method (PUM) [20]; it was chosen because of its capability to model crack initiation and propagation without remeshing and because it does not need to conform to internal boundaries. This method, originally introduced by Belytschko and Black [21], required a minimal remeshing to model crack initiation and propagation. It was later modified by Moës et al. [22], introducing a finite element method for crack growth that did not need remeshing in order to propagate. A Heaviside function was adopted to enrich the field throughout the length of the crack, in order to extend the required discontinuity along the crack length. Near the crack tip, enrichment functions, derived from linear elastic fracture mechanics (LEFM), were used to enrich the field in that zone. XFEM was also extended to the modeling of 3D crack propagation problems [23] and Giner et al. [24] implemented the XFEM capability into ABAQUS software [25]. In this paper, XFEM is used to trigger the onset of discrete failure and cracking, the three different failure criteria considered for the flat bars are implemented in the ABAQUS user subroutine 
UDMGINI, which is connected to the XFEM ABAQUS ability.

This work has introduced a new methodology to model crack propagation in ductile and brittle materials. This is achieved by using a failure criterion to trigger crack initiation and using localization analysis (loss of ellipticity) to predict the crack propagation. The direction of the crack is predicted by choosing appropriate plasticity formulation for the material and using the normal and polarization vectors of the discontinuity surface.

\section{Theoretical background}

\subsection{Kirsch's elastic stress concentration problem}

In his seminal paper on circular perforations, Kirsch [26] presented the linear elastic stress distribution at a point $\mathrm{P}$ (see Figure 1) in form of equation (1) taken from [27]. Starting from a perforated circular sheet under uniform tension and using polar coordinates he presented the solution for finite flat bars under axial tension. Elementary mechanics of materials text books show that the stress concentration factor for a circular hole in an infinite plate is equal to 3. However this factor for a finite plate is a function of the ratio of the hole diameter to the width of the plane and approaches 2 when the diameter of the hole increases.

$$
\begin{aligned}
\sigma_{r r} & =\frac{\sigma}{2}\left(1-\frac{a^{2}}{r^{2}}\right)+\frac{\sigma}{2}\left(1-\frac{a^{2}}{r^{2}}\right)\left(1-\frac{3 a^{2}}{r^{2}}\right) \cos (2 \theta) \\
\sigma_{\theta \theta} & =\frac{\sigma}{2}\left(1+\frac{a^{2}}{r^{2}}\right)-\frac{\sigma}{2}\left(1+\frac{3 a^{4}}{r^{4}}\right) \cos (2 \theta) \\
\sigma_{r \theta} & =-\frac{\sigma}{2}\left(1-\frac{a^{2}}{r^{2}}\right)\left(1+\frac{3 a^{2}}{r^{2}}\right) \sin (2 \theta)
\end{aligned}
$$




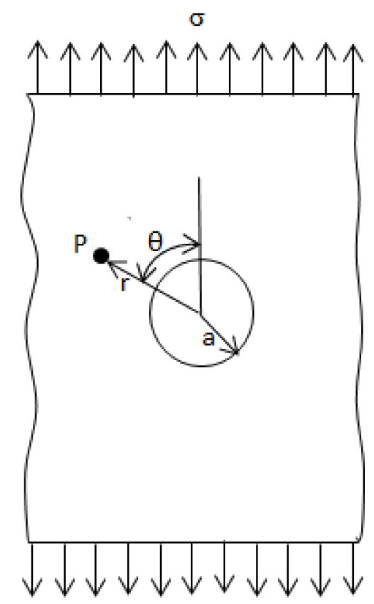

Figure 1: Schematics of a circular opening in an infinite sheet.

The starting point of the Kirsch solution was the linear elastic result of an infinite sheet with a circular hole under uniform tension in the far field. It should be noted that the classical stress concentration factor of 3 was re-evaluated with higher order continuum theories and gradient elasticity theories resulting in a significant reduction of the classical stress concentration factor 3 to values close to 2 .

Aside from the analytical stress concentration results of linear elasticity, Kirsch [26] presented in his paper the results of a series of experiments on cast iron and on mild steel flat bars ('Gusseisen und Flusseisen') with circular perforations of different diameters, in order to illustrate the effect of the hole size on the ultimate load capacity and tensile strength of cast iron and mild steel. In fact, the original paper was slightly critical of the use of linear elasticity theory for technical applications in view of his observations and experimental findings on the strength of the perforated flat bars tested 
in tension.

In his experimental tests, based on the linear elastic solution reported in equation (1), he expected to see an elastic stress concentration factor of 3 at the ligament edge of the circular perforation of the cast iron bar. However he found a reduction of less that fifteen percent, in contradiction with the linear elastic solution governing brittle fracture initiation at $1 / 3$ of the unperforated strength of cast iron flat bars.

This raises the question whether strain hardening, localized yielding and plastic deformations may explain this contradictory behavior.

In the case of mild steel, the experimental results of Kirsch exhibit no notch sensitivity of the load bearing capacity of the tested specimen. This can be explained by the high ductility and plastic redistribution capacity of the material due to hardening in the region near stress concentrations near the ligament edges of the circular perforation.

\subsection{Extended finite element method}

In the most general case of XFEM, the displacement approximation is described as:

$$
\mathbf{u}_{x f e m}(\mathbf{x})=\sum_{i=\phi} N_{i}(\mathbf{x}) \cdot \mathbf{u}_{i}+\sum_{i=\phi_{H}} N_{i}(\mathbf{x}) \cdot H(\mathbf{x}) \cdot \mathbf{a}_{i}+\sum_{i=\phi_{C}} N_{i}(\mathbf{x}) \cdot \sum_{\alpha=1}^{4} F_{\alpha}(\mathbf{x}) \cdot \mathbf{b}_{i \alpha}
$$

where $\phi$ is the set of all nodes in the far field domain, $\phi_{H}$ is the set of nodes containing the crack and $\phi_{C}$ is the set of nodes containing the crack tip. $H$ is the Heaviside function, $F_{\alpha}$ are the crack-tip functions, $\mathbf{a}_{i}$ and $\mathbf{b}_{i}$ are the degrees of freedom of the enriched nodes.

As shown in Figure 2 the crack does not have to follow the element edges and 
it could pass through the elements. For simplicity here it is assumed that all the elements are fully cracked, i.e., there is no need to consider crack-tip enrichment functions (see Figure 3); in this way the complexity of the problem is reduced while the accuracy of the model is preserved.

From equation (3) it is concluded that the physical displacement at an enriched node does not correspond to the true displacement, therefore the shifted basis formulation is introduced as a modification to the formulation, expressed as:

$$
\mathbf{u}_{x f e m}(\mathbf{x})=\sum_{i=\phi} N_{i}(\mathbf{x}) \cdot \mathbf{u}_{i}+\sum_{i=\phi_{H}} N_{i}(\mathbf{x}) \cdot\left[H(\mathbf{x})-H\left(\mathbf{x}_{i}\right)\right] \cdot \mathbf{a}_{i}
$$

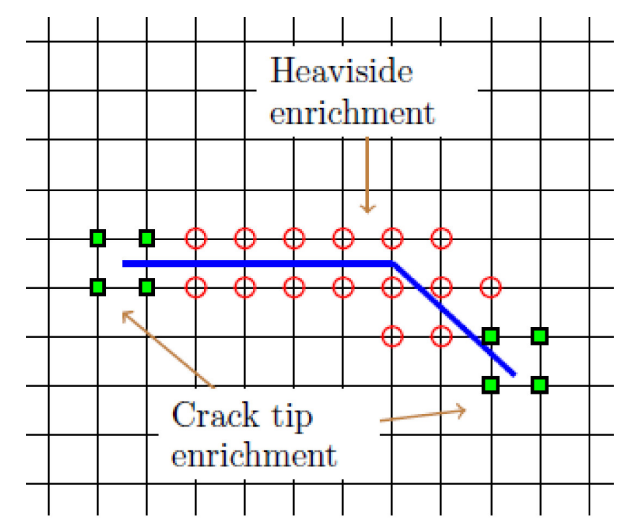

Figure 2: Heaviside and crack-tip function. 

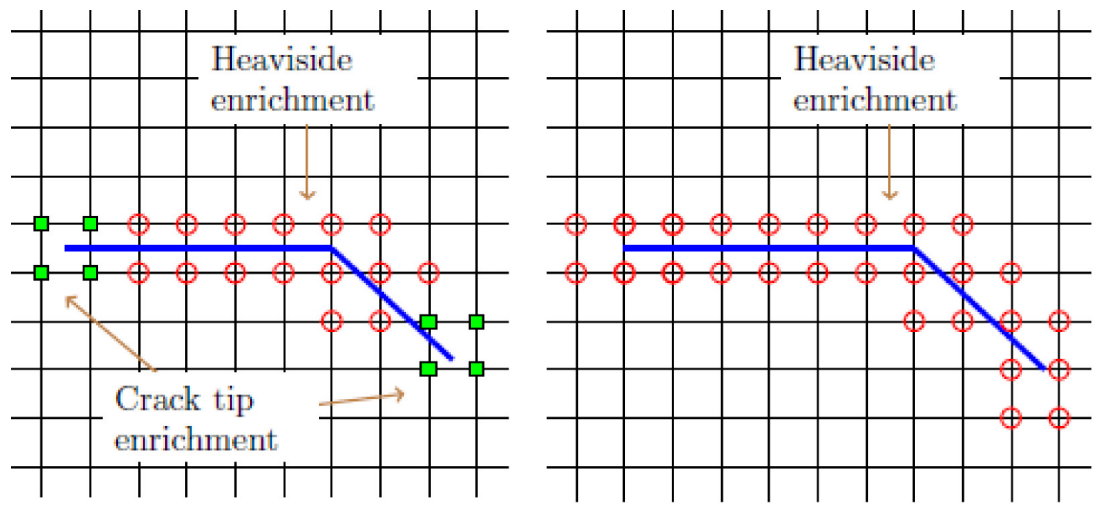

Figure 3: Modification of the enrichment by removing crack-tip enrichments.

In this work it is assumed that the material remains continuous, until a certain fracture initiation criterion is met and then the crack grows in the direction that has the critical localization properties. The XFEM capability of ABAQUS software is used while the initiation and propagation of the crack is controlled by a user subroutine called UDMGINI [25].

ABAQUS has few capabilities of extended finite element method modelling, among which the cohesive segment method with phantom nodes is here chosen. This method assumes a traction-separation cohesive behavior for the element that can be used for both brittle and ductile material behaviors. Phantom nodes are imposed on the original nodes and are constrained to the original nodes when the element is continuous, however, when the element is cracked, the element splits into two parts where each part contains a combination of real and phantom nodes (see Figure 4).

The element is assumed to be totally cracked when the cohesive strength of the element goes to zero and the phantom and real nodes move independently. 


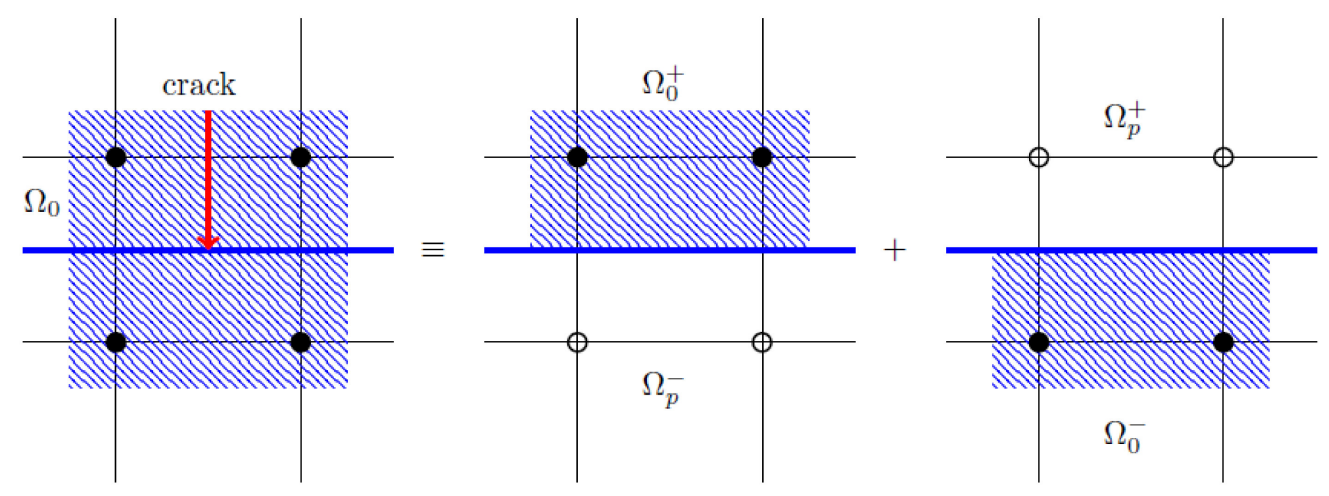

Figure 4: Phantom nodes.

In order to have a set of full interpolation basis, the part of the real domain of the cracked element, $\Omega_{0}$, is extended to the phantom domain, $\Omega_{p}$, and therefore the displacement in the real domain, $\Omega_{0}$, can be interpolated by the nodes of the phantom and real domain. The jump in the displacement field is calculated by only integrating the real part of each domain i.e., $\Omega_{0}^{+}$ and $\Omega_{0}^{-}$. The traction-separation behavior used in this study is based on a linear uncoupled material model expressed as:

$$
\mathbf{t}=\left(\begin{array}{c}
t_{n} \\
t_{s} \\
t_{t}
\end{array}\right)=\left(\begin{array}{ccc}
K_{n n} & 0 & 0 \\
0 & K_{s s} & 0 \\
0 & 0 & K_{t t}
\end{array}\right) \otimes\left(\begin{array}{c}
\delta_{n} \\
\delta_{s} \\
\delta_{t}
\end{array}\right)=\mathbf{K} \otimes \boldsymbol{\delta}
$$

where the units of $K_{i i}$ are pressures divided by displacement.

The implementation of the user subroutine requires the introduction of a failure criterion and a normal vector defining the direction of cracking. The failure criteria used in this work are the strain format of $J_{2}$ plasticity, Tresca and St. Venant in the form of a normalized formulation which produces a value between zero and one. The crack propagation occurs when this criterion 
is equal to one. The direction of the crack is predicted by the localization analysis of the failure criteria. As an example, in the case of cast iron, the Rankine criterion is used and the localization analysis predicts that the crack propagates perpendicular to the direction of the major principal stress.

\subsection{Localization Analysis}

In this section localized failure (or loss of ellipticity) is discussed, analytical localization analysis is performed and the critical angles and hardening values associated with Rankine, von Mises and Tresca criteria are presented [17].

\subsubsection{Loss of Ellipticity}

While diffuse failure refers to a material instability and zero or negative values of the second order work density, localized failure, in form of weak discontinuities, reflects the formation of jumps of the strain rates across merging surfaces while the rate of the displacements field remains continuous across the discontinuity surface:

$$
\begin{aligned}
& {[[\dot{\mathbf{u}}]]=\dot{\mathbf{u}}^{+}-\dot{\mathbf{u}}^{-}=0,} \\
& {[[\nabla \dot{\mathbf{u}}]]=\nabla \dot{\mathbf{u}}^{+}-\nabla \dot{\mathbf{u}}^{-} \neq 0 .}
\end{aligned}
$$

The "+" and "-" signs refer to the positive and negative sides of the discontinuity surface. In order to satisfy the Maxwell compatibility condition, the jump in the gradient of the displacement field should have the form:

$$
[[\nabla \dot{\mathbf{u}}]]=\dot{\gamma} \mathbf{M} \otimes \mathbf{N}
$$


where the scalar value $\dot{\gamma}$ expresses the magnitude of the jump across the discontinuity surface, $\mathbf{N}$ is the normal to this surface and $\mathbf{M}$ is the polarization vector which defines the direction of the jump i.e., if it is parallel to $\mathbf{N}$ it represents an opening mode, if it is perpendicular to $\mathbf{N}$ the jump is a tangential shear mode, and other angles indicate a mixed mode jump condition of shear strain rate.

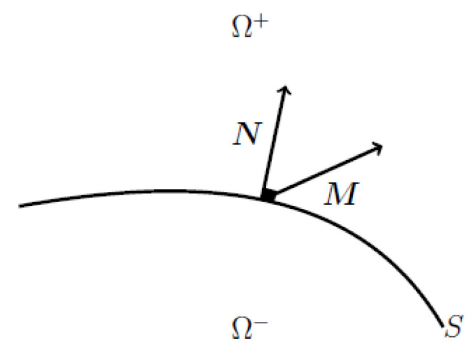

Figure 5: Discontinuity surface.

The discontinuity in the strain rate field is defined as,

$$
[[\nabla \boldsymbol{\epsilon}]]=[[\nabla \dot{\mathbf{u}}]]=\frac{1}{2} \dot{\gamma}(\mathbf{M} \otimes \mathbf{N}+\mathbf{N} \otimes \mathbf{M}) .
$$

In order to maintain equilibrium across the discontinuity surface, it is required that the traction vectors on both sides of the discontinuity should be equal and in opposite direction, i.e.,

$$
[[\dot{\mathbf{t}}]]=\dot{\mathbf{t}}^{+}-\dot{\mathbf{t}}^{-}=0 .
$$

According to Cauchy's lemma, this leads to the localization condition here reported, 


$$
\begin{aligned}
& {[[\dot{\mathbf{t}}]]=\mathbf{N} \cdot[[\dot{\boldsymbol{\sigma}}]]=\mathbf{N} \cdot\left[\left[\mathbf{E}^{\mathbf{e p}}: \dot{\boldsymbol{\epsilon}}\right]\right]=\mathbf{N} \cdot \mathbf{E}^{\mathbf{e p}}:[[\dot{\boldsymbol{\epsilon}}]] } \\
\Rightarrow & {[[\dot{\mathbf{t}}]]=\dot{\gamma} \mathbf{N} \cdot \mathbf{E}^{\mathbf{e p}} \cdot \mathbf{N} \cdot \mathbf{M}=\dot{\gamma} \mathbf{Q}^{\mathbf{e p}} \cdot \mathbf{M}=0 }
\end{aligned}
$$

where $\mathbf{Q}^{\mathbf{e p}}$ is the elastoplastic second order localization tensor, defined as follows:

$$
\mathrm{Q}^{\mathrm{ep}}=\mathrm{N} \cdot \mathbf{E}^{\mathrm{ep}} \cdot \mathbf{N}
$$

Therefore, the loss of ellipticity happens when the elastoplastic localization tensor (acoustic tensor) is singular, i.e.,

$$
\operatorname{det}\left(\mathbf{Q}^{\mathbf{e p}}\right)=0 \Rightarrow \lambda_{\min }\left(\mathbf{Q}^{\mathbf{e p}}\right)=0
$$

Based on equation (11), the loss of ellipticity occurs when the minimum eigenvalue of the acoustic tensor is equal to zero.

The elastoplastic localization tensor may be expressed as:

$$
\mathrm{Q}^{\mathrm{ep}}=\mathbf{Q}^{\mathrm{e}}-\frac{\mathbf{N} \cdot \mathbf{E}^{\mathrm{e}}: \mathbf{m} \otimes \mathbf{n}: \mathbf{E}^{\mathrm{e}} \cdot \mathbf{N}}{H^{p}}
$$

Based on Ottosen [28], the eigenvalue problem is so defined:

$$
\operatorname{det}\left[\left(\mathbf{Q}^{\mathbf{e p}}\right)^{-\mathbf{1}} \cdot \mathbf{Q}^{\mathbf{e p}}\right]=0
$$

where

$$
\left(\mathbf{Q}^{\mathbf{e}}\right)^{-\mathbf{1}} \cdot \mathbf{Q}^{\mathbf{e p}}=\mathbf{1}-\left(\mathbf{Q}^{\mathbf{e}}\right)^{-\mathbf{1}} \cdot \frac{\mathbf{N} \cdot \mathbf{E}^{\mathrm{e}}: \mathbf{m} \otimes \mathbf{n}: \mathbf{E}^{\mathrm{e}} \cdot \mathbf{N}}{H^{p}} .
$$

Therefore the solution of the lowest eigenvalue of equation (14) becomes 


$$
\lambda_{\min }\left[\left(\mathbf{Q}^{\mathbf{e}}\right)^{-\mathbf{1}} \cdot \mathbf{Q}^{\mathrm{ep}}\right]=\mathbf{1}-\frac{\mathbf{N} \cdot \mathbf{E}^{\mathbf{e}}: \mathbf{m} \cdot\left(\mathbf{Q}^{\mathbf{e}}\right)^{-\mathbf{1}} \cdot \mathbf{n}: \mathbf{E}^{\mathbf{e}} \cdot \mathbf{N}}{H^{p}+\mathbf{n}: \mathbf{E}^{\mathbf{e}}: \mathbf{m}}
$$

and the critical hardening modulus indicating loss of ellipticity may be expressed as:

$$
H_{p}^{c r i t}=\mathbf{n}: \mathbf{E}^{\mathbf{e}} \cdot \mathbf{N} \cdot\left(\mathbf{Q}^{\mathrm{e}}\right)^{-\mathbf{1}} \cdot \mathbf{N} \cdot \mathbf{E}^{\mathbf{e}}: \mathbf{n}-\mathbf{n}: \mathbf{E}^{\mathbf{e}}: \mathbf{m} .
$$

\subsubsection{Localization analysis for different yield functions}

The analytical solution of the localization analysis, for different elastoplastic models adopted in order to characterize the behavior of the ferrous flat bars, is presented in this subsection. The localization analysis can be performed for the case of associated and non-associated plasticity; here the attention is focused on the associated one. The critical angle is defined as the angle between the first principal axis of the normal to the yield surface, $n_{1}$, and the normal to the localization surface. This angle, in the case of associated plasticity, is on the plane 1-3 (see Figure 6 a)) and is defined as:

$$
\tan ^{2}\left(\theta_{c r}\right)=-\frac{n_{3}+\nu \cdot n_{2}}{n_{1}+\nu \cdot n_{2}}
$$

where $n_{i=1,3}$ are the eigenvalues of the normal to the yield surface. In the case of non-associated flow rule, the normal to the discontinuity surface has components in all the directions. Therefore, based on Figure 6 b), the components of the normal to the discontinuity surface become:

$$
N_{1}=\cos \phi \cdot \cos \alpha ; \quad N_{2}=\cos \phi \cdot \sin \alpha ; \quad N_{3}=\sin \phi
$$




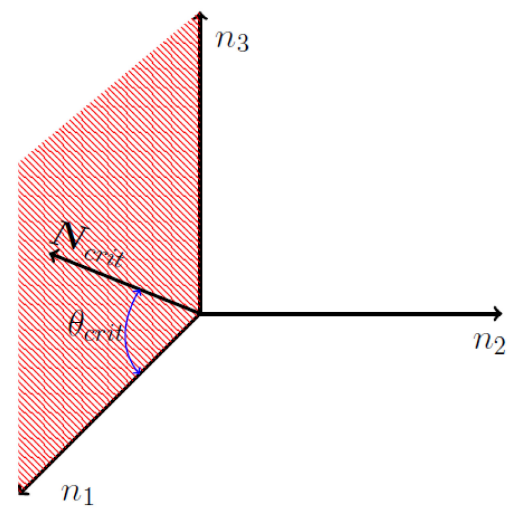

a)

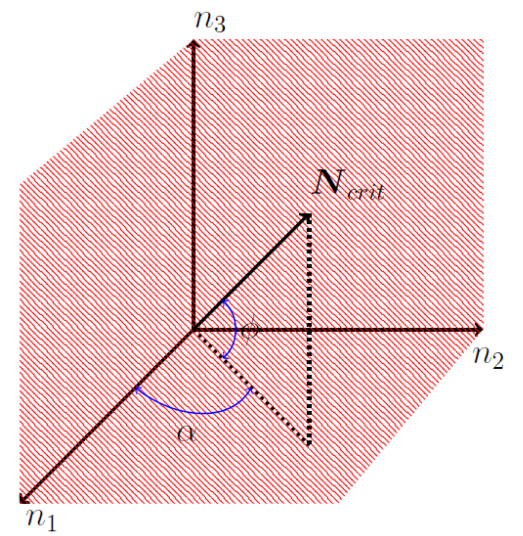

b)

Figure 6: Normal to the discontinuity surface: a) associated, b) non associated flow rule.

As regards to the loading direction, the corresponding critical angle is shown in Figure 7.
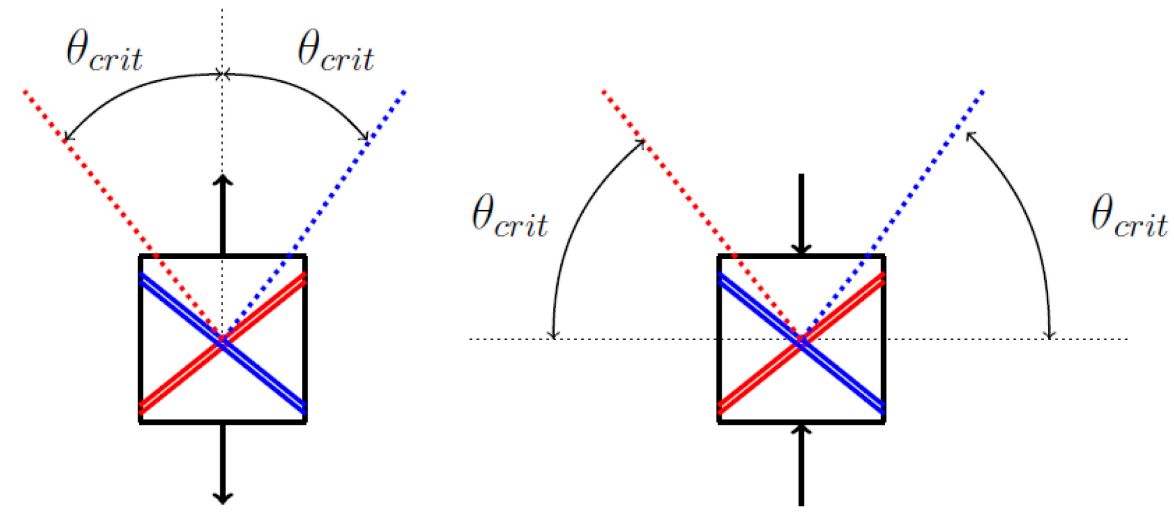

Figure 7: Critical angle $\theta_{\text {crit }}$ in tension and compression.

Rankine Criterion. This criterion, mostly used for brittle material behavior, assumes that yielding occurs when the principal stress reaches the value $\sigma_{y 0}$ 
and it is described as:

$$
F=\sigma_{1}-\sigma_{y 0} .
$$

The normal to the yield surface becomes:

$$
\mathbf{n}=\mathbf{m}=\frac{\partial F}{\partial \sigma}=\left(\begin{array}{lll}
1 & 0 & 0 \\
0 & 0 & 0 \\
0 & 0 & 0
\end{array}\right) .
$$

Consequently the critical angle, $\theta_{c r}$, and the critical hardening, $H_{c r}$, assume the values below

$$
\begin{gathered}
\tan ^{2}\left(\theta_{c r}\right)=0 \rightarrow\left\{\begin{array}{l}
\theta_{c r, 1}=0 \\
\theta_{c r, 2}=0
\end{array},\right. \\
H_{c r}=0
\end{gathered}
$$

This confirms the modes of failure associated with Rankine's criterion, i.e. the material fails perpendicular to the direction of the major principal stress (see Figure 8). 


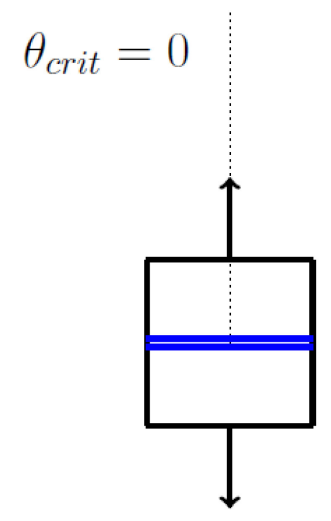

Figure 8: Critical angle and corresponding failure surface, Rankine Criterion.

von Mises Criterion. This criterion, with linear isotropic hardening, depends on the second invariant of the deviatoric stress tensor and it is described as:

$$
F=3 \sqrt{J_{2}}-\sigma_{y}(K)=0
$$

and the normal to the yield surface takes the following form:

$$
\mathbf{n}=\frac{\partial F}{\partial \sigma}=\frac{\sqrt{3}}{2 \sqrt{J_{2}}}\left(\begin{array}{ccc}
\frac{2 \sigma_{1}-\sigma_{2}-\sigma_{3}}{3} & 0 & 0 \\
0 & \frac{2 \sigma_{2}-\sigma_{1}-\sigma_{3}}{3} & 0 \\
0 & 0 & \frac{2 \sigma_{3}-\sigma_{1}-\sigma_{2}}{3}
\end{array}\right) .
$$

Finally, based on equation (17), the critical angle $\theta_{c r}$ and the critical hardening $H_{c r}$ values are here presented:

$$
\begin{gathered}
\tan ^{2}\left(\theta_{c r}\right)=-\frac{s_{3}+\nu \cdot s_{2}}{s_{1}+\nu \cdot s_{2}} \\
H_{c r}=-\frac{3 E \cdot s_{2}{ }^{2}}{2 \cdot\left(s_{1}{ }^{2}+s_{2}{ }^{2}+s_{3}{ }^{2}\right)} .
\end{gathered}
$$


Tresca Criterion. The criterion is defined as:

$$
F=\left|\frac{\sigma_{1}-\sigma_{3}}{2}\right|-\tau_{\max }=0
$$

therefore the normal to the yield function becomes:

$$
\mathbf{n}=\frac{\partial F}{\partial \sigma}=\frac{1}{2}\left(\begin{array}{ccc}
1 & 0 & 0 \\
0 & 0 & 0 \\
0 & 0 & -1
\end{array}\right) .
$$

This results in the critical angle, $\theta_{c r}$, and the critical hardening, $H_{c r}$, values below

$$
\begin{gathered}
\tan ^{2}\left(\theta_{c r}\right)=1 \rightarrow\left\{\begin{array}{l}
\theta_{c r, 1}=+45^{\circ} \\
\theta_{c r, 2}=-45^{\circ}
\end{array},\right. \\
H_{c r}=0 .
\end{gathered}
$$

As expected, the critical angles associated with Tresca are $45^{\circ}$, with respect to the principal directions; that is in agreement with the pure shear failure mode of ductile materials.

\section{Experimental Tests}

Several perforated and unperforated flat bar specimens made of mild steel, high strength steel, cast iron and aluminum were prepared based on ASTM standards and were tested under uniaxial tension. Ten coupons were tested for each material type and duplicate coupons for each hole diameter, that varies from a $1 / 16$ to $1 / 4$ of an inch. The geometry of these bars is depicted in Figure 9 a), while their chemical composition is reported in Table 1. 
Table 1: Chemical composition of the tested flat bars.

\begin{tabular}{|c|c|c|c|c|c|c|c|c|c|c|}
\hline & & $\mathrm{C}$ & Mn & $\mathbf{P}$ & $\mathbf{S}$ & $\mathrm{Si}$ & $\mathrm{Ni}$ & Mo & $\mathrm{Cr}$ & $\mathrm{Cu}$ \\
\hline \multicolumn{2}{|l|}{ Cast Iron } & 3.09 & 0.76 & 0.022 & 0.19 & 2.69 & 0.04 & $<0.01$ & 0.08 & 0.07 \\
\hline \multicolumn{2}{|l|}{ Mild Steel } & 0.16 & 0.41 & 0.01 & 0.004 & 0.045 & 0.07 & 0.01 & 0.08 & 0.24 \\
\hline \multicolumn{2}{|c|}{ High Strength Steel } & 0.21 & 0.66 & 0.009 & 0.012 & 0.20 & 0.07 & 0.01 & 0.13 & 0.16 \\
\hline & $\mathrm{Al}$ & \multicolumn{2}{|l|}{ V } & $\mathrm{Ti}$ & $\mathrm{Nb}$ & Co & B & W & $\mathrm{Zr}$ & Sn \\
\hline Cast Iron & 0.01 & \multicolumn{2}{|c|}{$<0.01$} & $<0.01$ & $<0.01$ & 0.008 & 0.0005 & $<0.01$ & $<0.01$ & 0.06 \\
\hline Mild Steel & 0.023 & \multicolumn{2}{|c|}{$<0.002$} & $<0.002$ & $<0.002$ & 0.007 & $<0.005$ & $<0.01$ & $<0.01$ & 0.009 \\
\hline High Strength Steel & 0.006 & \multicolumn{2}{|c|}{$<0.002$} & $<0.002$ & $<0.002$ & 0.007 & $<0.005$ & $<0.01$ & $<0.01$ & 0.006 \\
\hline $\mathrm{Si}$ & $\mathrm{Fe}$ & \multicolumn{2}{|l|}{$\mathrm{Cu}$} & Mn & $\mathrm{Mg}$ & \multicolumn{2}{|l|}{$\mathrm{Cr}$} & $\mathrm{Zn}$ & $\mathrm{Ti}$ & Other \\
\hline Al 6061 & $<0.7$ & \multicolumn{2}{|c|}{$0.15-0.4$} & $<0.15$ & $0.8-1.2$ & \multicolumn{2}{|c|}{$0.04-0.35$} & 0.25 & 0.15 & 0.15 \\
\hline
\end{tabular}

The experiments were performed under displacement control and the rate of the displacement controlled test is kept the same for all the test samples, in order to eliminate any possible strain rate effect. The test setups adopted are a Tinius Olsen and a Shore Western test frame (see this last frame in Figure 9 b)) and a photogrammetric non-contact device (digital image correlation system) was used in order to provide additional data in the form of displacement and strain field on the surface of the specimens [14]. The observations of this DIC system were compared with the LVDT measurements in order to study the accuracy of the device.

The flat bars were placed inside the grips of the test setup and the photogrammetric device was mounted in front of the specimen according to the instructions of the GOM optical measuring techniques company. The specimens were painted in white and then black speckles were sprayed on the specimen. The speckle pattern of a painted cast iron specimen is depicted 
in Figure 9 c). Two cameras were placed in front of the specimen in order to provide a 3D measurement field; these cameras observe the deformation of the specimen through the images captured at time intervals by various rectangular image details known as facets. These facets are quadrilaterals that include a portion of the specimen in form of pixels and cover $n \times n$ pixels of the specimen that are equally distanced from one another. The 2D coordinates of the facets are calculated based on the corners points of the quadrilateral and the centre of the facet is calculated by averaging the four corners. Using the information from left and right cameras, the 3D coordinates of the facet points are calculated.

The ultimate load capacity and strength of each material is obtained and the effect of perforation on the ductility, strength and load capacity of that material is assessed and compared. Modes of failure and fracture based on the material behavior are then explained and the transition of brittle-ductile failure is studied. 


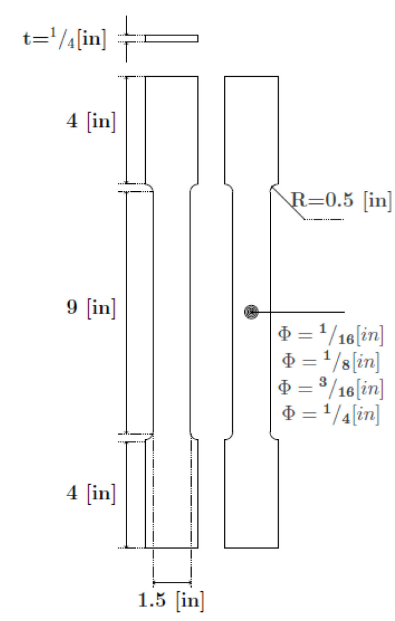

a)

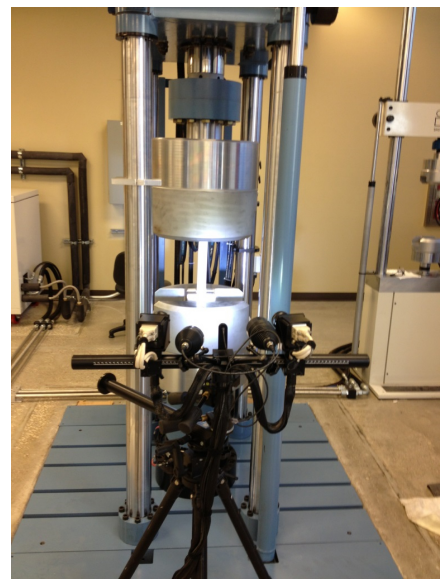

b)

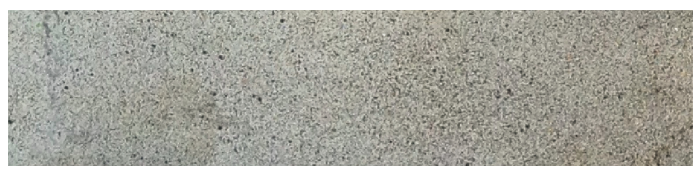

c)

Figure 9: a) Dimension of the flat bars; b) test setup and c) speckle pattern on the painted specimen.

\subsection{Failure Mechanisms}

Having all the specimens carefully painted, the tension tests are performed and the failed specimens are shown in Figure 10.

Metals failure and fracture can be described by three mechanisms: ductile fracture, cleavage and inter-granular fracture; in specific the first two are the most common mechanisms. 


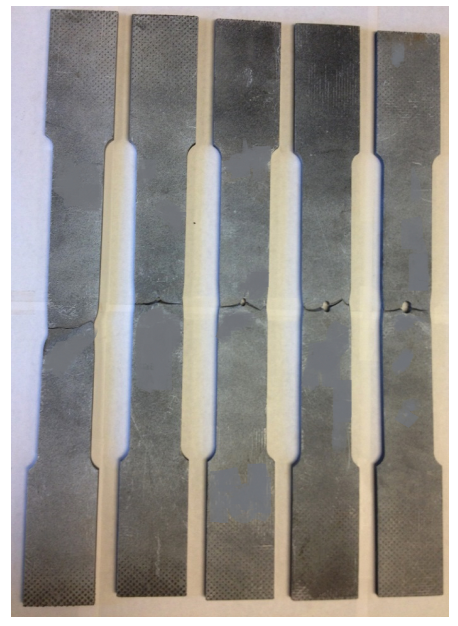

a)

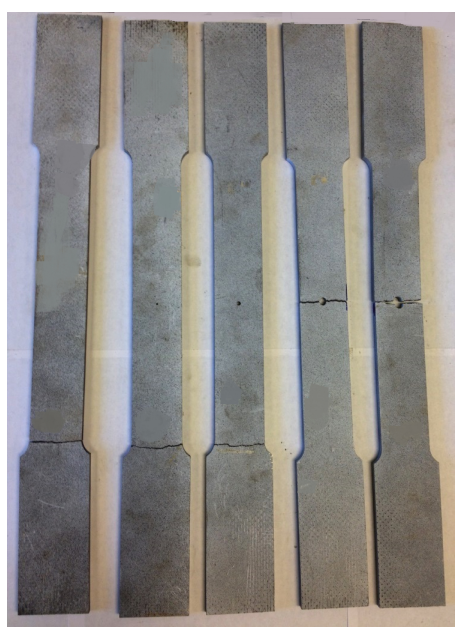

c)

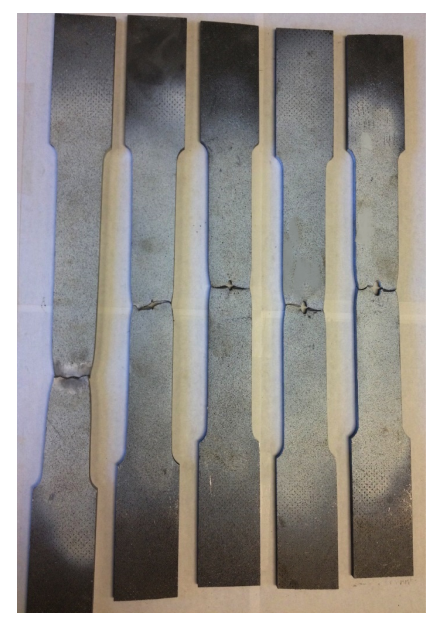

b)

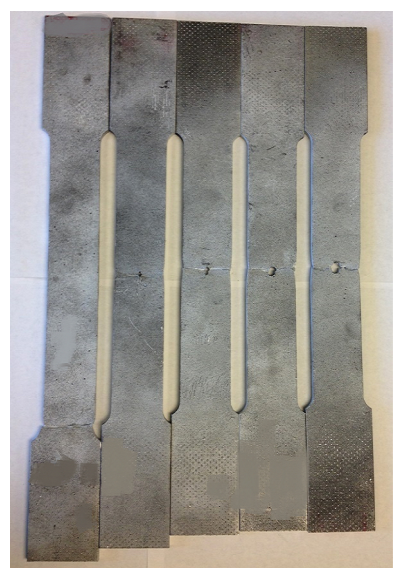

d)

Figure 10: Failed flat bars under uniaxial tension test: a) high strength steel; b) mild steel; c) cast iron; d) aluminum.

Ductile fracture consists of three stages: formation of a free surface at an inclusion or second phase material; void growth because of hydrostatic 
stress and the coalescence of voids. Void nucleation and coalescence are the main causes of failure at the centre of the specimen because of the presence of a triaxial state of stress and an higher density of voids; however, due to a lower density of the voids and the shear dominant state of stress close to the edges, the material exhibits a shear dominant failure close to the free edges. This explains the failure of mild steel specimens, while in the case of the aluminum, the failure is a shear dominant failure with an inclined outof-plane failure plane. In the case of high strength steel the failure is also a mixed mode of void coalescence and shear failure. It should be noted that since the thickness of the specimens are relatively small in compare to their width, the out-of-plane failure mechanism is a shear failure.

In the case of perforated specimens, the cracks tend to grow in the direction of the maximum equivalent strain while the global constraints force the cracks to remain perpendicular to the direction of loading; the presence of these constraints form a zig-zag pattern as described by Anderson [29], that results in a cleavage failure mechanism of the core material.

Cleavage failure mechanism is the rapid propagation of a crack in a transgranular pattern. This is the most common failure mechanism for brittle metals, however it should be noted that it can be preceded by ductile and plastic behavior. The plane of failure is perpendicular to the direction of loading and, for a brittle behavior, the failure can be explained by the major principal stress. This failure mechanism perfectly describes the failure of the cast iron specimens.

Cleavage failure of cast iron flat bars and shear failure of mild steel and high strength steel flat bars are depicted in Figure 11. 


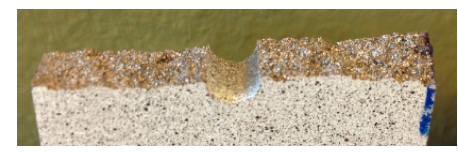

a) Cast Iron

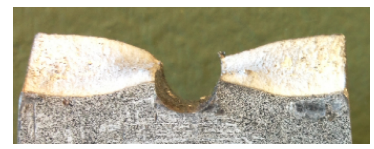

b) High Strength Steel

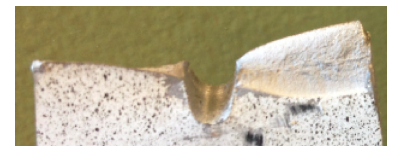

c) Mild Steel

Figure 11: Through-the-thickness failure of flat bars.

\subsection{Experimental Observations}

The load-deformation response curves of the tested flat bars of mild steel, aluminum, high strength steel and cast iron are reported in Figure 12.

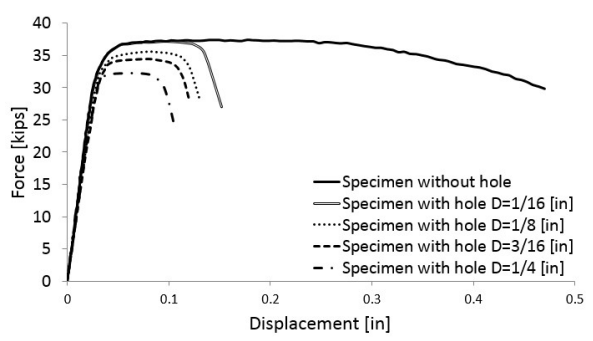

a)

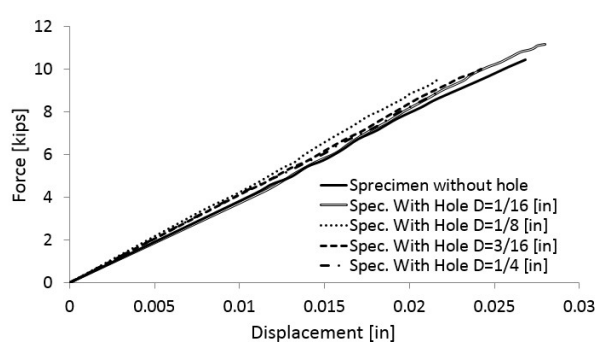

c)

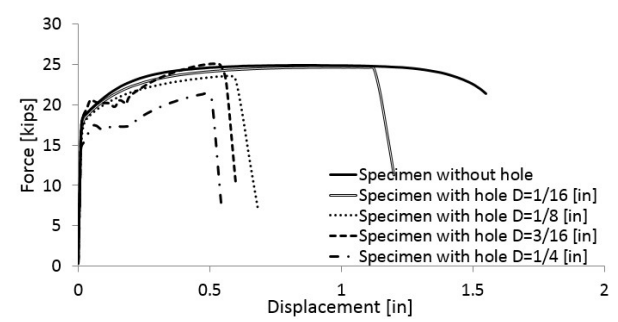

b)

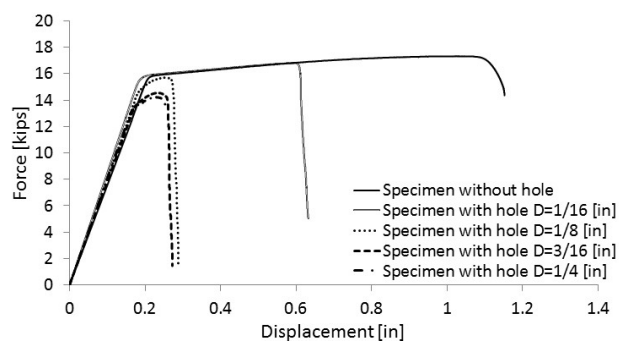

d)

Figure 12: Load-deformation curves of flat bars: a) high strength steel; b) mild steel; c) cast iron; d) aluminum alloy. 
Measuring the load-deformation curves, it is possible to obtain the stressstrain curves of the flat bars and consequently plot the change in the ultimate load capacity and ultimate strength of these specimen with respect to the circular perforation diameter. In order to compare different materials, the nominal strength or load capacity of the notched specimens are normalized by the ultimate strength or load capacity of the unnotched specimen. As a result of the circular perforation, it is expected that the ultimate load capacity of the perforated specimens decreases to a third of the unperforated specimens. However, from Figure 13, it can be observed that the reduction is only about the $15 \%$. It can be also concluded that, although the ultimate load capacity of the specimens decreases by increasing the size of the hole, the ultimate tensile strength of those specimens does not change drastically.

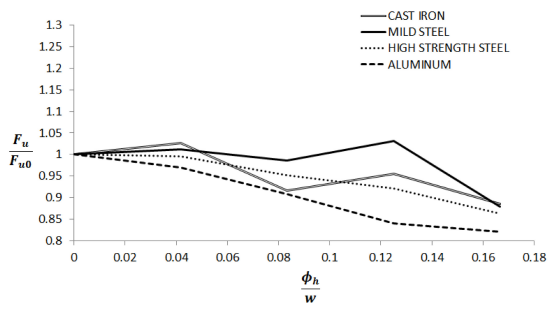

a)

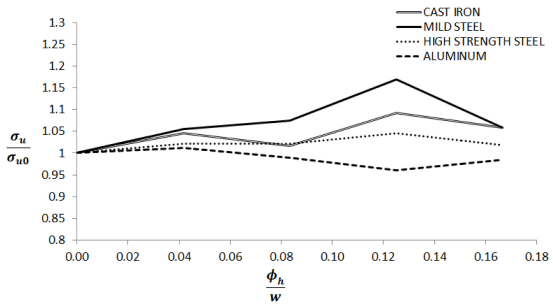

b)

Figure 13: a) Ultimate load ratio and b) ultimate stress ratio of the perforated specimens.

\subsection{Digital Image Correlation Observations}

The displacement field and strain field over the entire region of interest are captured and analysed using a digital image correlation system (DIC). This photogrammetric non-contact device is mounted in front of the painted sample, for continuous recording of the relative movement of black dots on 
the white specimen during the deformation.

In order to validate the readings of the device, a series of experiments are performed on cast iron flat bars. Having the DIC system configured and calibrated, the test is started using displacement control and both the DIC system and the LVDT start to collect the data simultaneously.

After the test is over, the displacements are measured, using the post processing toolbox in ARAMIS software, in the vicinity of the area where the LVDT is mounted and are compared to the LVDT results (see Figure 14: Test 1, refers to the sample without perforation while the rest are for the samples with different hole sizes; where Test 2 is for the smallest hole size and Test 5 for the largest).

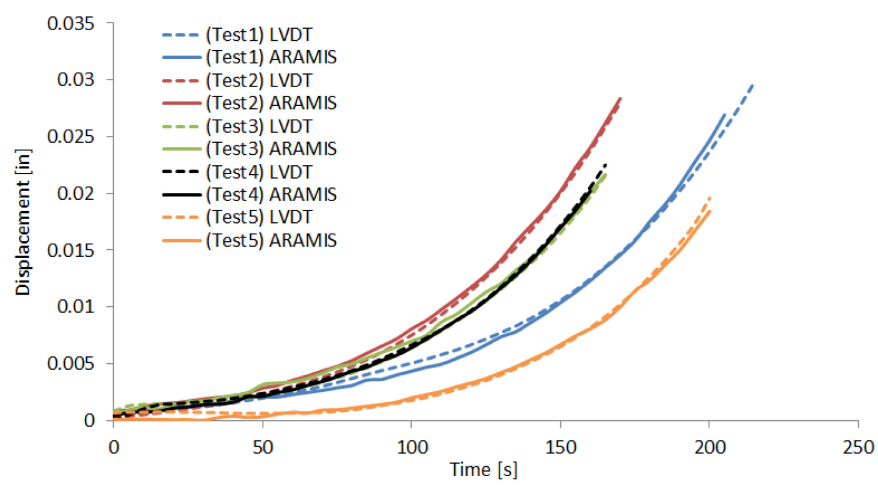

Figure 14: Comparison of LVDT and ARAMIS data.

Based on the experimental data the root mean square deviation is calculated and presented in Table 2 . 
Table 2: Root mean square deviation of ARAMIS and LVDT.

\begin{tabular}{cccccc} 
Test & 1 & 2 & 3 & 4 & 5 \\
\hline Error[in] & $5.05 \mathrm{e}-4$ & $4.84 \mathrm{e}-4$ & $5.76 \mathrm{e}-4$ & $2.42 \mathrm{e}-4$ & $3.98 \mathrm{e}-4$
\end{tabular}

From the root mean square deviation calculation it can be observed that the difference between the LVDT and the DIC measurements is negligible. The comparison of the longitudinal strain distribution in the vicinity of the perforation, with a diameter of $1 / 4$ inch for mild steel, aluminum, high strength steel and cast iron is shown in Figure 15. The figures clearly show the presence of shear (Lüder) bands.
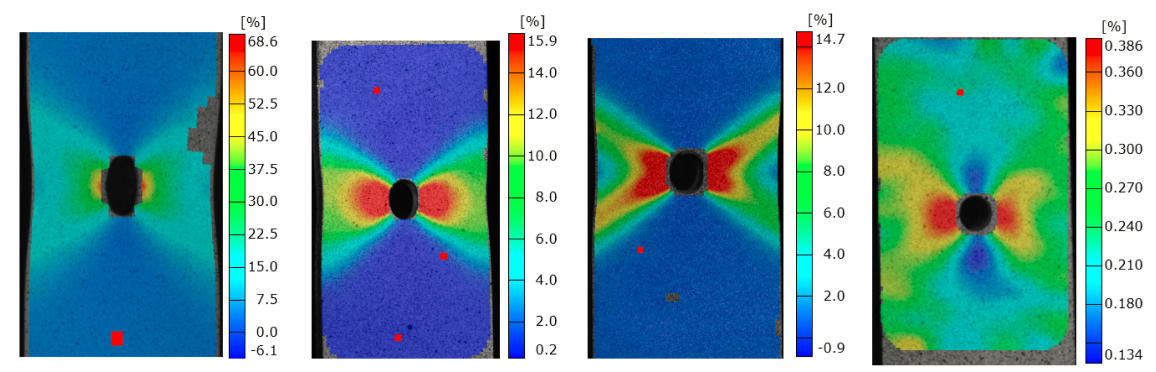

Figure 15: Longitudinal strain field for mild steel, aluminum, high strength steel and cast iron.

\section{Simulation of metallic specimens}

This section is dedicated to the numerical results obtained for metallic specimens, starting with the calibration of the numerical models proposed for mild steel, aluminum, high strength steel and cast iron based on the ex-

perimental observations performed on flat bars. Afterwards the concept of 
inelastic XFEM is introduced, by coupling localization analysis and the extended finite element method, and the ductile and brittle behavior of these specimens are examined and investigated by acquiring different failure criteria and localization properties.

In order to simulate the behavior of the metallic specimens, the experimental data of the unperforated specimens are extracted and used for the calibration of the proposed models. These models include the von Mises isotropic hardening/softening plasticity formulation for mild steel, high strength steel and aluminum and the St. Venant criterion for cast iron. These criteria are used in order to trigger the onset of plastification for the ductile specimens and the onset of brittle failure for cast iron. However, for the case of ductile materials, another criterion has to be satisfied before the material behavior becomes unstable and crack growth rapidly ruptures the specimen; this criterion is expressed in terms of the strain invariants since it is preceded by plastification. In summary, two criteria are required for ductile failure, the first one in terms of stresses to account for plasticity and the second one in terms of strains to initiate cracking, while for brittle failure one criterion is sufficient and it could be either in terms of strains or stresses.

Finally the direction of the failure is predicted based on localization analysis of the failure criteria. It should be noted that in this work localization analysis of the strain format of the von Mises and Tresca criteria is considered for ductile materials while for brittle cast iron localization properties of the St. Venant or Rankine criteria are used.

A step-by-step description of the simulation algorithm in the form of a flowchart is reported in Figure 16. 


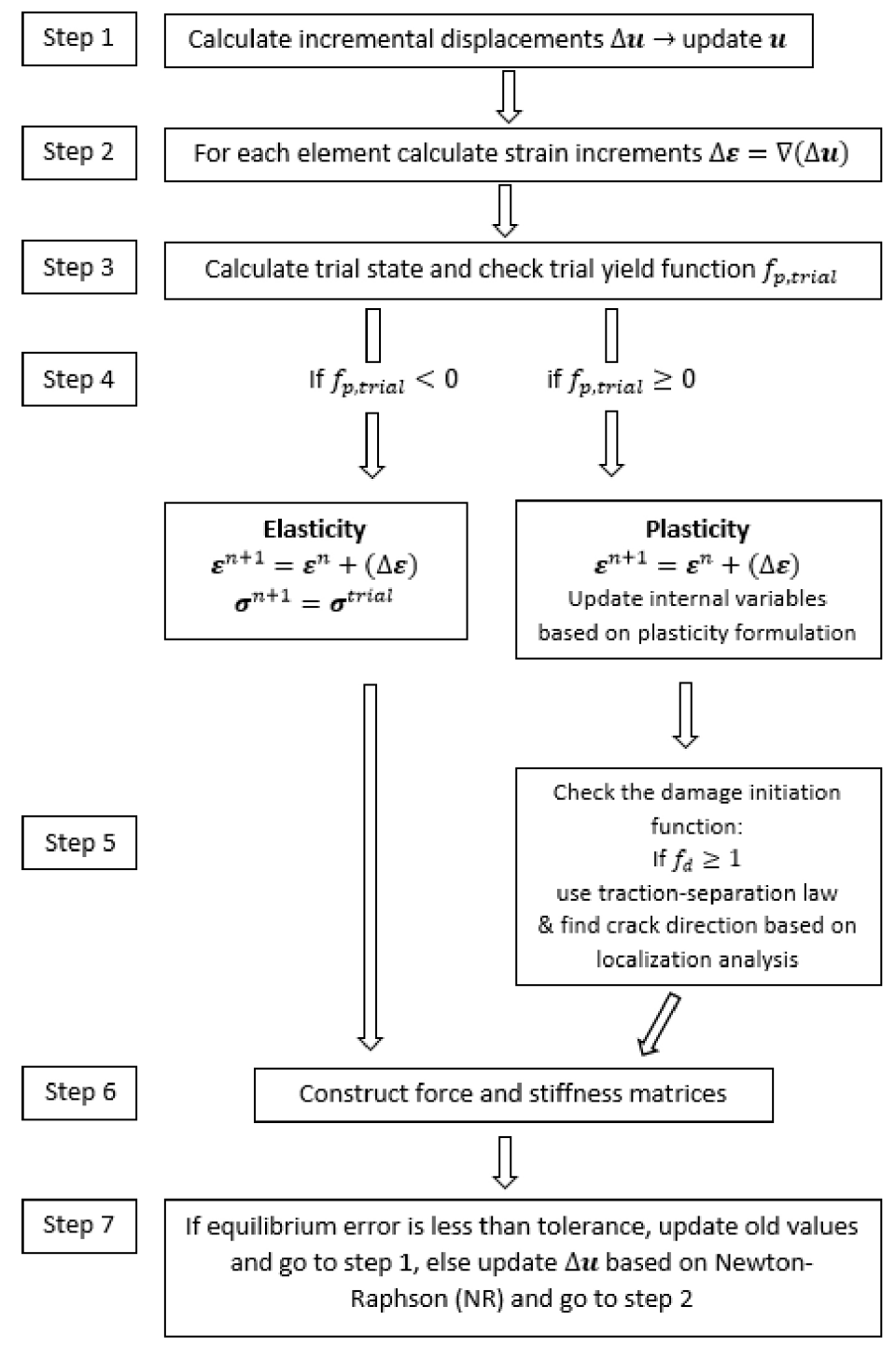

Figure 16: Step-by-step description of the simulation algorithm.

\subsection{Calibration of the numerical models}

In the case of mild steel linear elastic behavior is assumed up to yielding, the von Mises plasticity with isotropic hardening coupled with extended 
finite element method and localization analysis is used to capture the post yield behavior and this model is calibrated with the experimental results obtained from the non-perforated experiments. The comparison of the calibrated model and experimental results is depicted in Figure 17.

The stress values that are used for calibration are the true stress values and are obtained as:

$$
\sigma^{\text {true }}=\left(1+\epsilon^{\text {nom }}\right) \cdot \sigma^{\text {nom }}
$$

The corresponding load-deformation curve of the calibrated models for the different materials are depicted respectively in Figure 17, Figure 18 and Figure 19 .

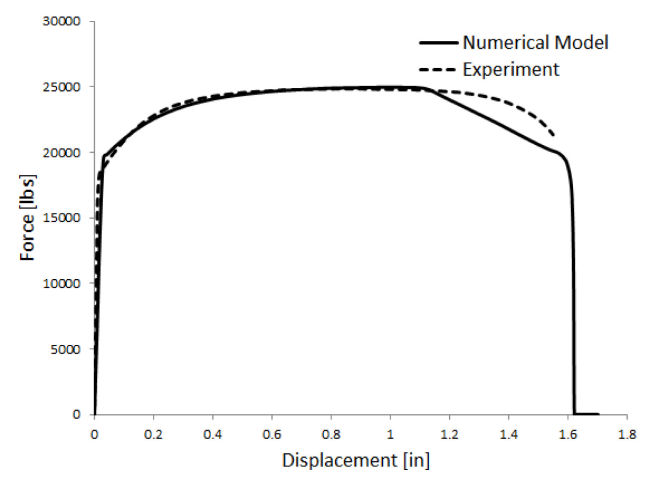

Figure 17: Comparison of the load-deformation curve of the experiment and numerical model for mild steel. 


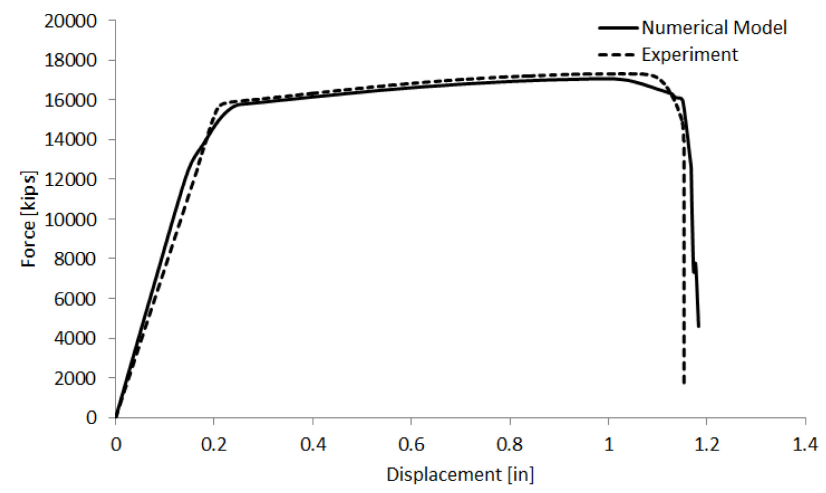

Figure 18: Comparison of the load-deformation curve of the experiment and numerical model for aluminum.

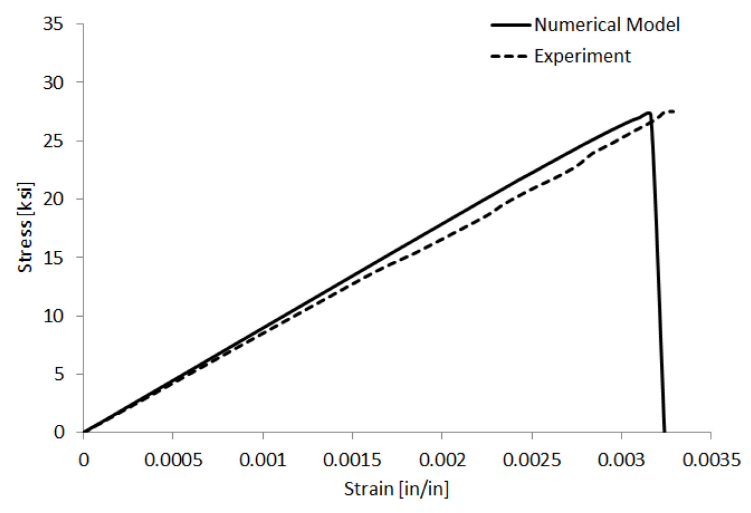

Figure 19: Comparison of the stress-strain curve of the experiment and numerical model for cast iron.

\subsection{Analysis of the failure direction}

In this part three different failure criteria are implemented in the ABAQUS subroutine UDMGINI [25] in order to trigger the onset of discrete failure and cracking. Since cast iron exhibits brittle behavior, Saint Venant or Rankine criteria are used for the initiation of the failure, and the localization analysis of these criteria results in a failure direction that is perpendicular to 
the direction of the major principal stress or strain. A comparison of the experimental observations and numerical models for the perforated cast iron specimen is presented in Figure 20.
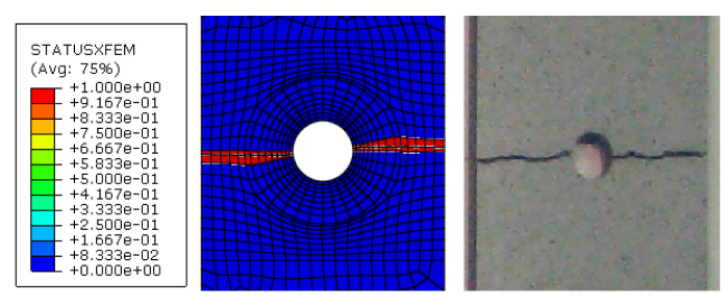

Figure 20: Comparison of the XFEM and experimental result for cast iron.

In the case of ductile materials, Tresca and von Mises criteria are used to predict the failure directions.

For Tresca criterion, as seen previously in this work, the critical angles are determined to be \pm 45 degrees with respect to the principal directions of stress. This predicts that the normal to failure surface is inclined of 45 degrees in the plane of $n_{1}-n_{3}$ and, based on the direction of the $n_{3}$ vector, this surface could be inclined on the surface and perpendicular to the direction of loading in the out-of-plane direction or it could be inclined in the out-ofplane direction and normal to the direction of the loading in the plane. The failure surface based on the second case of the $n_{1}-n_{3}$ surface is presented in Figure 21. 


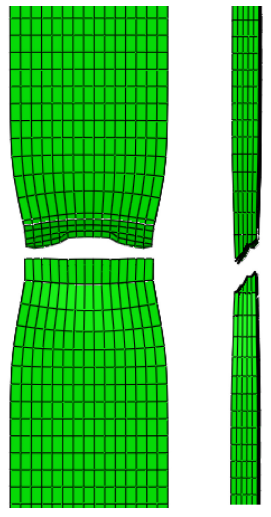

Figure 21: Failure surface based on localization analysis of Tresca criterion.

For von Mises criterion, the localization analysis provides different results for an associated in compare to a non-associated plasticity model. Furthermore, this model has different critical angles based on the type of loading and whether the model is a $3 \mathrm{D}$ model or $2 \mathrm{D}$ plane stress or plane strain. The results of localization analysis for the associated case are summarized in Table 3, where $f(\nu)$ is defined as

$$
\theta_{\text {crit }}= \pm \arctan \sqrt{-\frac{n_{3}+\nu n_{2}}{n_{1}+\nu n_{2}}}
$$

Table 3: Critical angles of associated von Mises plasticity based on localization analysis.

\begin{tabular}{lcccc}
\hline & Tension & Compression & Shear & BiaxialTension \\
\hline Plane Stress $\boldsymbol{\theta}_{\text {crit }}\left[^{\circ}\right]$ & 35.26 & 54.74 & 45.00 & 0.00 \\
\hline Plane Strain $\boldsymbol{\theta}_{\text {crit }}\left[^{\circ}\right]$ & 45.00 & 45.00 & 45.00 & 45.00 \\
\hline Three Dimensional $\left[^{\circ}\right]$ & $f(\nu)$ & $f(\nu)$ & $f(\nu)$ & $f(\nu)$ \\
\hline
\end{tabular}

The comparison of the inelastic XFEM modelling in the case of associated von Mises plasticity for the case of plane stress and plane strain under 
compressive loading is depicted in Figure 22. The critical angle for plane stress case under compression is 54.74 degrees with respect to the direction of major principal stress and this angle is 45 degrees for the case of plane strain.
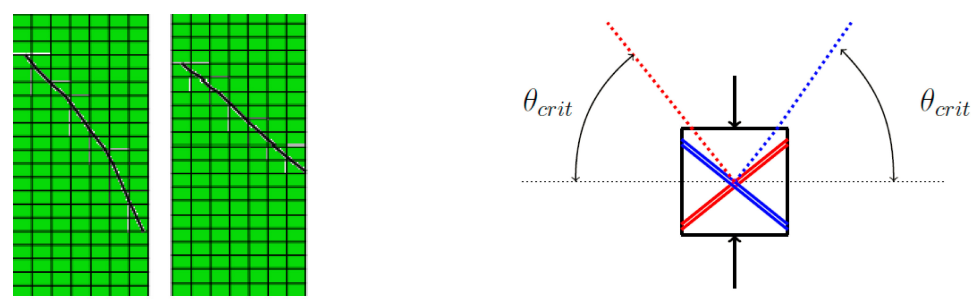

Figure 22: Critical angle for plane stress and plane strain under compression for associated von Mises plasticity

As an example, for the case of three dimensional modelling and uniaxial tension, the critical angle for von Mises associated plasticity is calculated as:

$$
\boldsymbol{s}=\left(\begin{array}{c}
\frac{2 \sigma}{3} \\
-\frac{\sigma}{3} \\
-\frac{\sigma}{3} \\
0 \\
0 \\
0
\end{array}\right) ; \quad \mathbf{n}=\frac{\sqrt{3} \boldsymbol{s}}{2 \sqrt{J_{2}}} \rightarrow \mathbf{n}=\left(\begin{array}{c}
1 \\
-\frac{1}{2} \\
-\frac{1}{2} \\
0 \\
0 \\
0
\end{array}\right) \rightarrow \tan ^{2}\left(\theta_{\text {crit }}\right)=\frac{1+\nu}{2-\nu}
$$

In this work the general case of associated von Mises plasticity localization analysis is used to find the critical angle of localization. For this reason at every step of loading, for each element and Gauss point the principal values and directions of the normal to the yield function are calculated and based on that the critical angle is calculated and it is multiplied by a rotation matrix 
to transform it to the global coordinates. In the case that the equivalent von Mises strain reaches the critical value, the crack is initiated and will propagate in the direction perpendicular to the critical angle, where this angle is on the $n_{1}-n_{3}$ plane as shown in Figure 23.

$$
\text { if } \quad \mathrm{F}=\frac{\epsilon_{\mathrm{eq}}}{\epsilon_{\mathrm{c}}} \quad \text { then } \quad \theta_{\text {crit }}= \pm \arctan \sqrt{-\frac{\mathrm{n}_{3}+\nu \mathrm{n}_{2}}{\mathrm{n}_{1}+\nu \mathrm{n}_{2}}}
$$

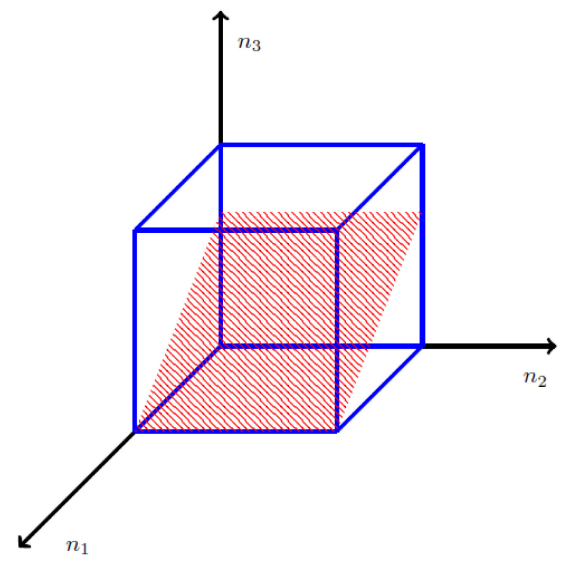

Figure 23: Crack surface.

Failure Direction, Rankine. Based on the Rankine criterion, the normal to the localization surface is parallel to the direction of major principal stress. The localization analysis of this model is used to investigate the behavior of the brittle cast iron flat bars. The boundary conditions and loading are designed in a manner to simulate the experimental tests. The failure direction of the perforated cast iron specimen is presented in Figure 20.

Failure Direction, Tresca. Tresca criterion is adopted in order to model the behavior of ductile material and it predicts that the critical angle of the 
normal to the localization surface is 45 degrees inclined with regard to the major and minor principal stress directions, for all the loading conditions. It is so assumed a priori for initial investigation of the failure surface compared to von Mises, where this angle varies according to the loading conditions. If the $n_{1}-n_{3}$ surface is parallel to the front surface of the specimen, then the crack would be inclined in the surface of the specimen; if the $n_{1}-n_{3}$ surface is perpendicular to the surface of the specimen, the crack would be inclined through the depth of the specimen and it would be horizontal on the surface, as shown in Figure 24.

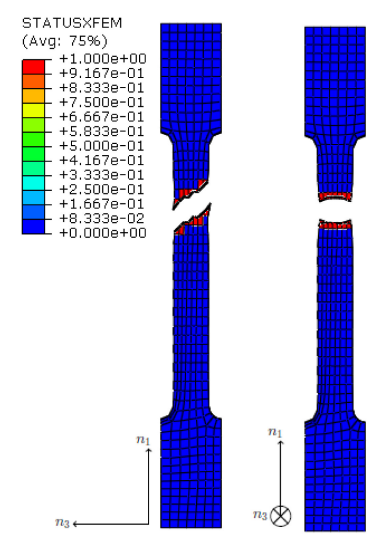

Figure 24: Comparison of the failure surface for different principal directions $n_{1}-n_{3}$.

Failure Direction, von Mises. The localization analysis of the associated von Mises plasticity is applied to the ductile flat bars in order to investigate the behavior of this model. The evolution of the von Mises stress and major principal strain distributions from the beginning of the test, onset of plastification, cracking and failure are depicted in Figure 25 and Figure 26. 


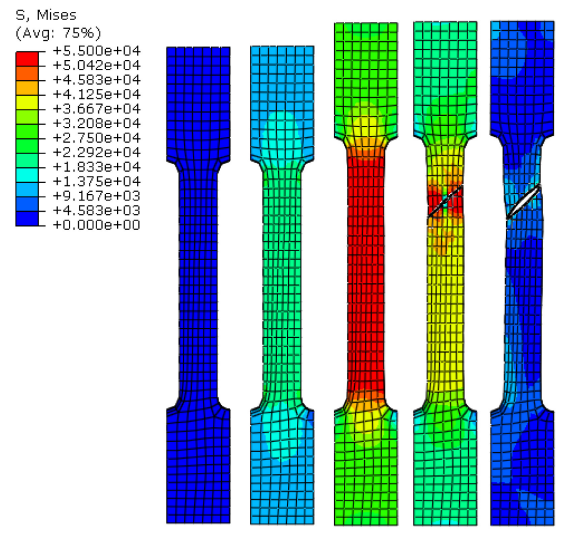

Figure 25: Distribution of the von Mises stress at different stages of loading.

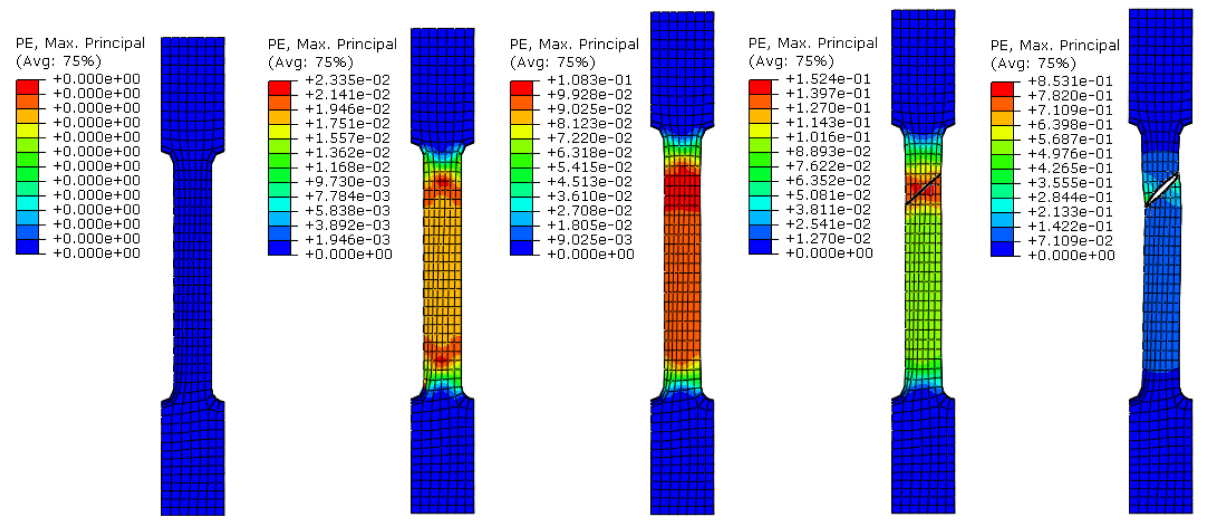

Figure 26: Distribution of the plastic strain at different stages of loading.

These results confirm the calculations of the localization analysis for the von Mises associated plasticity, which predicts that the crack is inclined with an angle approximately of 41 degrees. The directions of the major and minor principal stresses at the onset of cracking shows that the $n_{1}-n_{3}$ surface is parallel to the surface of the specimen and $n_{1}$ is in the direction of loading. The calibrated model is used for the perforated specimen and the evolution of the crack and plastic strain for the flat bar with $1 / 4$ circular diameter 
is depicted in Figure 27. A magnified view of the cracked surface for the perforated specimen is presented in Figure 28. It is observed that not only there is in-plane inclination of the crack surface in the $x-y$ plane, but also the crack is inclined in the $\mathrm{x}-\mathrm{z}$ plane.
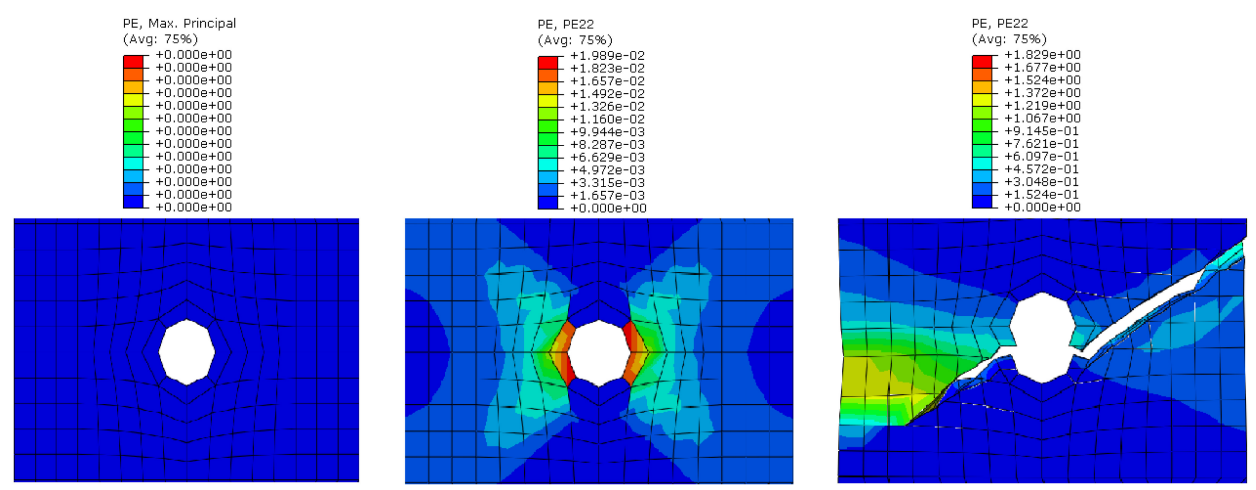

Figure 27: Particular of the distribution of the plastic strain at different stages of loading.

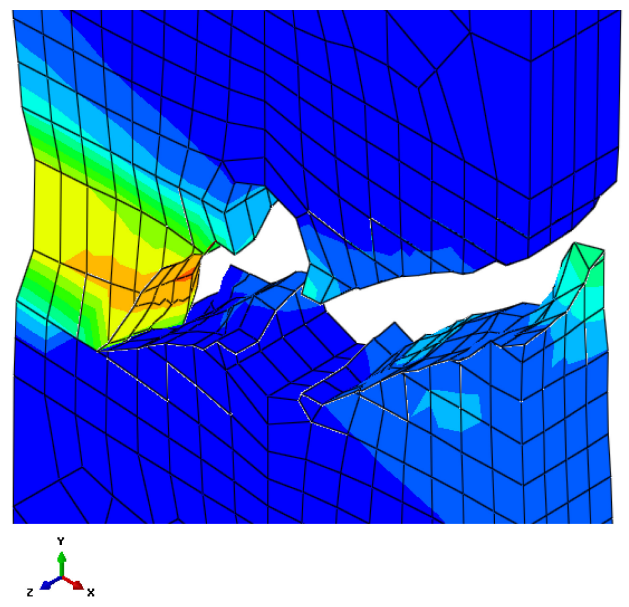

Figure 28: Isometric view of the cracked specimen. 


\section{Conclusions}

This paper addressed the unexpected reduction of the ultimate load bearing capacity due to stress concentration at the vicinity of a perforation and introduced a new methodology to follow crack propagation in ductile and brittle materials. In the first part, the load-deformation curves of the experimental observations on the perforated and non-perforated mild steel, aluminum, high strength steel and cast iron flat bars were obtained and, based on that, the effect of circular opening on the ultimate load capacity and tensile strength of these materials was investigated. Although it was expected to observe $\mathrm{a}^{2} / 3$ drop of the load capacity of the cast iron specimen because of the stress concentration effects, the load capacity of the perforated cast iron specimen only decreased by $15 \%$; this was also true for ductile materials where local plastification in the vicinity of the perforation was the main reason for this type of behavior. The result of these comparisons is depicted in Figure 13.

In the second part of this paper, different yield and failure criteria were studied and investigated in order to find an appropriate criteria for the onset of failure and the direction of crack propagation. It was observed that the Rankine and St. Venant criteria were capable of predicting the direction of crack propagation of the brittle cast iron specimen. Based on localization analysis, the normal to the failure surface was parallel to the principal stress/strain direction and therefore, the material failed perpendicular to this direction, which was in agreement with the experimental observations and is depicted in Figure 20.

In the case of the Tresca criterion it was observed that, if the direction of 
the minor principal stress of the normal to the yield surface was pointing through the thickness of the specimen, the failure surface predicted by this model was similar to the failure surface observed in the case of aluminum specimens. It should be noted that the critical angle based on this method was 45 degrees. This method was also a good prediction of the failure surface of the non-perforated mild steel that has an inclined failure surface in the out-of-plane direction and perpendicular to the direction of loading in the in-plane direction.

In the case of von Mises plasticity, the critical angle varied based on the associativity and non-associativity of the problem. In this paper it was assumed associated plasticity. The prediction of the normal to the failure surface for this model was different for $2 \mathrm{D}$ and $3 \mathrm{D}$ cases. It was observed that in the case of uniaxial tension for the plane stress case, this angle was 35.26 degrees and for the plane strain case it was 45 degrees. However in $3 \mathrm{D}$ cases, the critical angle was a function of the normal to the yield surface and Poisson's ratio. For the uniaxial 3D case this angle was approximately 41 degrees.

This confirmed the experimental results observed in the perforated mild steel and non-perforated high strength steel specimens as depicted in Figure 29. 


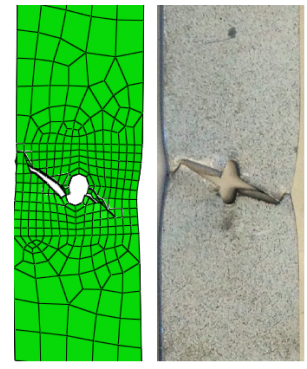

a)
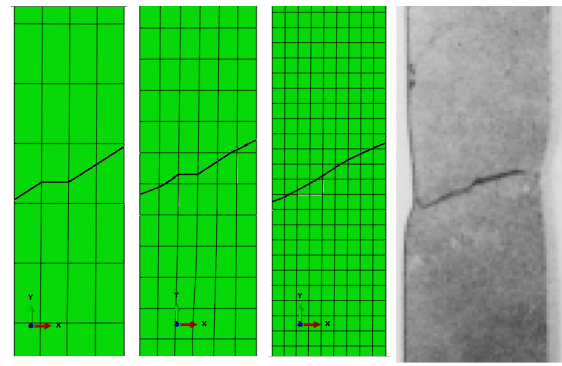

b)

Figure 29: Comparison of the failure mode predicted by von Mises criterion and the experimental results on: a) perforated mild steel and b) high strength steel.

In summary, this methodology which was a combination of extended finite element method and localization analysis was capable of predicting the onset of failure and crack propagation for different material behaviors. This methodology was investigated and studied for four materials by using different yield functions where it was shown that it could accurately model the crack propagation by using an appropriate failure criterion. This work could be further extended to study other materials with more sophisticated yield and failure criteria and would be a very helpful tool to predict the realistic failure and degradation of the material in sensitive and advanced industries.

\section{Acknowledgements}

The research was partially supported by the US National Science Foundation under NSF grant on 'Interface Mechanics of Masonry Panels under Biaxial Loading" to the University of Houston. Opinions expressed are those of the authors and do not reflect those of the sponsors. 


\section{References}

[1] Beizaee S, Willam K, Xotta G. The effect of circular openings on the brittle-ductile fracture of ferrous flat bars. FraMCoS-8, Toledo, Spain; 2013.

[2] Beizaee S. Constitutive Modeling and Numerical Implementation of Brittle and Ductile Material Behavior with the Aid of Inelastic XFEM and Damage-Plasticity Models. Ph.D. Dissertation. University of Houston; 2013.

[3] Tresca H. Mémoire sur l'écoulement des corps solides soumis à de fortes pressions. Comptes Rendus des Seances de l'Academie des Sciences, Paris; 1864, 59, p. 754-8.

[4] von Mises R. Mechanik der festen Körper im plastisch-deformablen Zustand. Nachrichten von der Gesellschaft der Wissenschaften zu Göttingen, Mathematisch-Physikalische Klasse 1913;1:582-92.

[5] Coulomb CA. Essai sur une application des règles de maximis et minimis à quelques problèmes de statique, relatifs à l'architecture. Mémoires de mathématique et de physique, présentés à l'Académie Royale des Sciences par divers savans; 1776, 7, p. 343-82.

[6] Drucker DC, Prager W. Soil mechanics and plastic analysis or limit design. Quarterly of Applied Mathematics 1952;10:157-65.

[7] Gurson AL. Continuum Theory of Ductile Rupture by Void Nucleation and Growth: Part I - Yield Criteria and Flow Rules for Porous Ductile 
Media. Journal of Engineering Materials and Technology 1977;99(1):215.

[8] Willam KJ, Warnke EP. Constitutive models for the triaxial behaviour of concrete. Proceedings of the International Association for Bridge and Structural Engineering 1975;19:1-30.

[9] Peters WH, Ranson WF. Digital Imaging Techniques in Experimental Stress Analysis. Opt Eng 1982;21(3):427-31.

[10] Peters WH, Ranson WF, Sutton MA, Chu TC, Anderson J. Application Of Digital Correlation Methods To Rigid Body Mechanics. Opt Eng 1983;22(6):738-42.

[11] Chu TC, Ranson WF, Sutton MA. Applications of Digital-ImageCorrelation Techniques to Experimental Mechanics. Experimental Mechanics 1985;25(3):232-44.

[12] Sutton MA, Cheng M, Peters WH, Chao YJ, McNeill SR. Application of an optimized digital correlation method to planar deformation analysis. Image and Vision Computing 1986;4(3):143-50.

[13] Sutton MA, McNeill SR, Helm JD, Chao YJ. Advances in TwoDimensional and Three-Dimensional Computer Vision. Topics in Applied Physics 2000;77:323-72.

[14] GOM mbH, ARAMIS User Manual - Software. GOM Optical Measurement Techniques, Braunschweig, Germany; 2011. 
[15] Willam K, Iordache MM. Computational Simulation of Discontinuous Failure Processes. Mecánica Computacional 1996;17:227-42.

[16] Kang HD, Willam KJ. Localization Characteristics of Triaxial Concrete Model. Journal of Engineering Mechanics 1999;125(8):941-50.

[17] Chaves EWV. A Three Dimensional Setting for Strong Discontinuities Modelling in Failure Mechanics. Ph.D. Dissertation. Universitat Politécnica de Catalunya; 2003.

[18] Rizzi E. Sulla localizzazione delle deformazioni in materiali e strutture. Tesi di Dottorato di Ricerca. Dipartimento di Ingegneria Strutturale, Politecnico di Milano, Milano, Italy; 1995.

[19] Rizzi E, Maier G, Willam K. On failure indicators in multidissipative materials. International Journal of Solids and Structures 1996;33(20$22): 3187-214$.

[20] Melenk JM, Babuška I. The partition of unity finite element method: Basic theory and applications. Computer Methods in Applied Mechanics and Engineering 1996;139:289-314.

[21] Belytschko T, Black T. Elastic crack growth in finite elements with minimal remeshing. International Journal for Numerical Methods in Engineering 1999;45(5):601-20.

[22] Moës N, Dolbow J, Belytschko T. A finite element method for crack growth without remeshing. International Journal for Numerical Methods in Engineering 1999;46(1):131-50. 
[23] Moës N, Sukumar N, Moran B, Belytschko T. An extended finite element method (X-FEM) for two- and three-dimensional crack modelling. European Congress on Computational Methods in Applied Sciences and Engineering; 2000.

[24] Giner E, Sukumar N, Tarancón JE, Fuenmayor FJ. An abaqus implementation of the extended finite element method. Engineering Fracture Mechanics 2009;76:347-68.

[25] ABAQUS, Version 6.12-1. Dassault Systemes Simulia Corporation. Providence, Rhode Island; 2012.

[26] Kirsch G. Die Theorie der Elastizität und die Bedürfnisse der Festigkeitslehre. Zeitschrift des Vereines Deutscher Ingenieure 1898;42:797-807.

[27] Boresi AP, Chong KP. Elasticity in engineering mechanics. $2^{\text {nd }}$ ed. New York: Wiley-Interscience; 2000.

[28] Ottosen NS. Properties of discontinuous bifurcation solutions in elastoplasticity. International Journal of Solids and Structures 1991;27:401-21.

[29] Anderson TL. Fracture Mechanics: Fundamentals and Applications, Vol. 2. CRC Press; 1995. 